\title{
Semi-Compatible and Weakly Compatible Self Maps in an Intuitionistic Fuzzy Metric Space
}

\author{
Harpreet Kaur ${ }^{1}$, Neha Ishesh Thakur $^{2}$, Saurabh Manro ${ }^{* 3}$ \\ ${ }^{1,2}$ Department of Mathematics, Desh Bhagat University, Mandi Gobindgarh, Punjab, India \\ ${ }^{3}$ School of Mathematics and Computer Applications, Thapar University, Patiala, Punjab, India
}

\begin{abstract}
The present paper is aimed to obtain a common fixed point theorem in intuitionistic fuzzy metric spaces under using the ideas of weak compatibility and semi compatibility for six self mappings. The results presented in this paper extended and generalized various known fixed point theorems in the literature in the setting of fuzzy and intuitionistic fuzzy metric spaces. As a consequence, the main results in this paper extend and unify many results in the topic of fixed points such as C. Alaca, D. Turkoglu and C. Yildiz, Fixed points in intuitionistic fuzzy metric spaces (Choas Solitons \& Fractals, 29(5)(2006), 1073-1078). Also, some corollaries of the main results are given.
\end{abstract}

Keywords: Common fixed point, Intuitionistic fuzzy metric space, Semi-compatible maps, Weak-compatible maps.

\section{INTRODUCTION}

The concept of fuzzy sets was introduced initially by Zadeh [1] in 1965. Kramosil and Michalek [2], George and Veeramani [3] modified the notion of fuzzy metric with the help of continuous t-norms. In 2004, Park [4] defined the notion of intuitionistic fuzzy metric space with the help of continuous $t$-norm and continuous $t$-conorm as a generalization of $G V$-fuzzy metric space. Alaca et al. [5], in 2006, redefined the notion of intuitionistic fuzzy metric space as a generalization of $K M$-fuzzy metric space. Turkoglu et al. [6] gave generalization of Jungck's common fixed point theorem [7] in intuitionistic fuzzy metric spaces. Recently, Singh and Chouhan [8] introduced the concept of compatible maps in fuzzy metric space and established a fixed point theorem for four self-mappings.

In this paper, we obtain a common fixed point theorem in intuitionistic fuzzy metric spaces using the concept of weak compatibility and semi compatibility for six self mappings. The results presented in this paper extended and generalized various known fixed point theorems in the literature in the setting of fuzzy and intuitionistic fuzzy metric spaces such as C. Alaca, D. Turkoglu and C. Yildiz, Fixed points in intuitionistic fuzzy metric spaces (Choas Solitons \& Fractals, 29(5)(2006), 1073-1078). Also, some corollaries of the main results are given.

\section{PRELIMINARIES AND DEFINITIONS}

Definition 2.1. [9]: A binary operation $*:[0,1] \times[0,1] \rightarrow[0,1]$ is said to be a continuous $t$-norm if $*$ satisfies the following conditions: for all $a, b, c, d \in[0,1]$,

(i) * is commutative and associative;

(ii) $a * 1=a$;

(iii) $a * b \leq c * d$ whenever $a \leq c, \quad b \leq d$;

(iv) $*$ is continuous.

Definition 2.2. [9]: A binary operation $\diamond:[0,1] \times[0,1] \rightarrow[0,1]$ is called a continuous $\boldsymbol{t}$-conorm if $\diamond$ satisfies the following conditions: for all $a, b, c, d \in[0,1]$,

(i) $\diamond$ is commutative and associative;

(ii) $\diamond$ is continuous;

(iii) $a \diamond 0=a$;

(iv) $a \diamond b \leq c \diamond d$ when ever $a \leq c$ and $b \leq d$.

*Corresponding Author: sauravmanro@hotmail.com 
Definition 2.3.[5]: A 5-tuple $(X, M, N, *, \diamond)$ is said to be an intuitionistic fuzzy metric space if $X$ is a non-empty set, $*$ is a continuous $t$-norm, $\diamond$ is a continuous $t$-conorm and $M, N$ are fuzzy sets on $X^{2} \times[0, \infty)$ satisfying the following conditions: for all $x, y, z \in X$ and $s, t>0$,

(i) $M(x, y, t)+N(x, y, t) \leq 1$;

(ii) $M(x, y, 0)=0$;

(iii) $M(x, y, t)=1$ if and only if $x=y$;

(iv) $M(x, y, t)=M(y, x, t)$;

(v) $M(x, y, t) * M(y, z, s) \leq M(x, z, t+s)$;

(vi) $M(x, y, \bullet):[0, \infty) \rightarrow[0,1]$ is left continuous;

(vii) $\lim _{t \rightarrow \infty} M(x, y, t)=1$;

(viii) $N(x, y, 0)=1$;

(ix) $N(x, y, t)=0$ if and only if $x=y$;

(x) $N(x, y, t)=N(y, x, t)$;

(xi) $N(x, y, t) \diamond N(y, z, s) \geq N(x, z, t+s)$;

(xii) $N(x, y, \bullet):[0, \infty) \rightarrow[0,1]$ is right continuous;

(xiii) $\lim _{t \rightarrow \infty} N(x, y, t)=0$.

Then $(M, N)$ is called an intuitionistic fuzzy metric on $X$. The functions $M(x, y, t)$ and $N(x, y, t)$ denote the degree of nearness and the degree of non-nearness between $x$ and $y$ w.r.t. $t$, respectively.

Definition 2.4.[5]: Let $(X, M, N, *, \diamond)$ be an intuitionistic fuzzy metric space. Then a sequence $\left\{x_{n}\right\}$ in $X$ is said to be:

(a) Cauchy sequence if for all $t>0$ and $p>0$,

$\lim _{n \rightarrow \infty} M\left(x_{n+p}, x_{n}, t\right)=1$ and $\lim _{n \rightarrow \infty} N\left(x_{n+p}, x_{n}, t\right)=0$.

(b) convergent to a point $x \in X$ if for all $t>0$,

$\lim _{n \rightarrow \infty} M\left(x_{n}, x, t\right)=1$ and $\lim _{n \rightarrow \infty} N\left(x_{n}, x, t\right)=0$.

Remark 2.1.[5]: Since $*$ and $\diamond$ are continuous $t$-norm and $t$-conorms, the limit determined from (vii) and (xiii) is unique.

Definition 2.5.[5]: An intuitionistic fuzzy metric space $\left(X, M, N,{ }^{*}, \diamond\right)$ is said to be complete if and only if every Cauchy sequence in $X$ is convergent.

Example 2.1.[6]: Let $X=[0,1]$ with the usual metric, $*$ be the continuous $t$-norm and $\diamond$ be the continuous $t$-conorm defined by $a * b=a b$ and $a \diamond b=\max \{a, b\}$ respectively, for all $a, b \in[0,1]$. For each $t \in(0, \infty)$ and $x, y \in X$, define $(M, N)$ by

$$
M(x, y, t)=\left\{\begin{array}{c}
\frac{t}{t+|x-y|}, t>0 \\
0, t=0
\end{array} \quad \text { and } N(x, y, t)=\left\{\begin{array}{c}
\frac{|x-y|}{t+|x-y|}, t>0 \\
1, t=0 .
\end{array}\right.\right.
$$


American Research Journal of Mathematics, Volume 1, Issue 2, April 2015

ISSN 2378-704X

Clearly, $(X, M, N, *, \diamond)$ is a complete intuitionistic fuzzy metric space.

Turkoglu et al. [6] extended the notion of compatible mappings to intuitionistic fuzzy metric spaces as follows:

Definition 2.6.[6]: A pair $(A, S)$ of self-mappings of an intuitionistic fuzzy metric space $(X, M, N, *, \diamond)$ is said to be compatible if

$\lim _{n \rightarrow \infty} M\left(A S x_{n}, S A x_{n}, t\right)=1$ and $\lim _{n \rightarrow \infty} N\left(A S x_{n}, S A x_{n}, t\right)=0$ for all $t>0$,

whenever $\left\{x_{n}\right\}$ is a sequence in $X$ such that $\lim _{n \rightarrow \infty} A x_{n}=\lim _{n \rightarrow \infty} S x_{n}=z$ for some $z \in X$.

Definition 2.7.[10]: A pair $(A, S)$ of self-mappings of an intuitionistic fuzzy metric space $(X, M, N, *, \diamond)$ is said to be semi-compatible if

$\lim _{n \rightarrow \infty} M\left(A S x_{n}, S z, t\right)=1$ and $\lim _{n \rightarrow \infty} N\left(A S x_{n}, S z, t\right)=0$ for all $t>0$,

whenever $\left\{x_{n}\right\}$ is a sequence in $X$ such that $\lim _{n \rightarrow \infty} A x_{n}=\lim _{n \rightarrow \infty} S x_{n}=z$ for some $z \in X$.

Definition 2.8.[5]: A pair $(A, S)$ of self-mappings of a metric space $(X, d)$ is said to be weakly compatible mappings if the mappings commute at all of their coincidence points, i.e., $A x=S x$ for some $x \in X$ implies $A S x=S A x$.

The proofs of our main results are based upon the following lemmas:

Lemma 2.1.[5]: Let $(X, M, N, *, \diamond)$ be an intuitionistic fuzzy metric space and $\left\{y_{n}\right\}$ be a sequence in $X$. If there exists a real number $k \in(0,1)$ such that

$M\left(y_{n}, y_{n+1}, k t\right) \geq M\left(y_{n-1}, y_{n}, t\right)$ and $N\left(y_{n}, y_{n+1}, k t\right) \leq N\left(y_{n-1}, y_{n}, t\right)$,

for all $t>0, n=0,1,2, \ldots$, then $\left\{y_{n}\right\}$ is a Cauchy sequence in $X$.

Lemma 2.2.[5]: Let $(X, M, N, *, \diamond)$ be an intuitionistic fuzzy metric space. If there exists a real number $k \in(0,1)$ such that

$M(x, y, k t) \geq M(x, y, t)$ and $\quad N(x, y, k t) \leq N(x, y, t)$ for all $x, y \in X, t>0$,

then $x=y$.

Lemma 2.3.[5]: In intuitionistic fuzzy metric space $(X, M, N, *, \diamond), M(x, y,$.$) is non-decreasing and N(x, y,$. is non-increasing for all $x, y \in X$.

Lemma 2.4.[11]: Let $A$ and $B$ be self-mappings from an intuitionistic fuzzy metric space $(X, M, N, *, \diamond)$ assume that $B$ is continuous. Then $(A, B)$ is semi-compatible if and only if $(A, B)$ is compatible.

\section{Main Results}

In this paper, we prove following common fixed point theorem for six self-mappings in intuitionistic fuzzy metric spaces.

Theorem 3.1: Let $A, B, S, T, P$ and $Q$ be self-mappings on a complete intuitionistic fuzzy metric space $(X, M, N, *, \diamond)$ satisfying

$P(X) \subset S T(X), Q(X) \subset A B(X)$,

$A B=B A, S T=T S, P B=B P$ and $Q T=T Q$,

either $P$ or $A B$ is continuous,

$(P, A B)$ is semi-compatible and $(Q, S T)$ is weak-compatible,

for all $x, y \in X$ and $t>0$

$M(P x, Q y, t) \geq r(\min \{M(S T y, P y, t), M(A B x, Q y, 2 t), M(A B x, S T y, t)\})$

and

$N(P x, Q y, t) \leq r^{\prime}(\max \{N(S T y, P y, t), N(A B x, Q y, 2 t), N(A B x, S T y, t)\})$ 
where $r:[0,1] \rightarrow[0,1]$ is a continuous increasing function, such that $r(t)>t$ for each $0<t<1$ and $r^{\prime}:[0,1] \rightarrow[0,1]$ is a continuous decreasing function, such that $r^{\prime}(t)<t$ for each $0<t<1$.

Then $A, B, S, T, P$ and $Q$ have a unique common fixed point in $X$.

Proof: Suppose $x_{0} \in X$, then from (3.1) there exists $x_{1}, x_{2} \in X$ such that $P x_{0}=S T x_{1}$ and $Q x_{1}=A B x_{2}$. In general, we can construct sequences $\left\{y_{n}\right\}$ and $\left\{x_{n}\right\}$ in $X$ such that

$y_{2 n}=P x_{2 n}=S T x_{2 n+1}$ and $y_{2 n+1}=Q x_{2 n+1}=A B x_{2 n+2}$ for $n=0,1,2 \ldots$

Put $x=x_{2 n}, y=x_{2 n+1}$ in (3.5), we get

$M\left(P x_{2 n}, Q x_{2 n+1}, \mathrm{t}\right) \geq r\left(\min \left\{M\left(S T x_{2 \mathrm{n}+1}, P x_{2 \mathrm{n}}, \mathrm{t}\right), M\left(A B x_{2 \mathrm{n}}, Q x_{2 n+1}, 2 t\right), M\left(A B x_{2 \mathrm{n}}, S T x_{2 n+1}, t\right)\right\}\right)$

and

$N\left(P x_{2 n}, Q x_{2 n+1}, t\right) \leq r^{\prime}\left(\max \left\{N\left(S T x_{2 n+1}, P x_{2 n}, t\right), N\left(A B x_{2 n}, Q x_{2 n+1}, 2 t\right), N\left(A B x_{2 n}, S T x_{2 n+1}, t\right)\right\}\right)$

This implies that

$M\left(y_{2 n}, y_{2 n+1}, t\right) \geq r\left(\min \left\{M\left(y_{2 n}, y_{2 n}, t\right), M\left(y_{2 n-1}, y 2 n+1,2 t\right), M\left(y_{2 n-1}, y_{2 n}, t\right)\right\}\right)$

$\geq r\left(\min \left\{1, M\left(y_{2 n-1}, y_{2 n}, t\right), M\left(y_{2 n}, y_{2 n+1}, t\right), M\left(y_{2 n-1}, y_{2 n}, t\right)\right\}\right)$

and

$N\left(y_{2 n}, y_{2 n+1}, t\right) \leq r^{\prime}\left(\max \left\{N\left(y_{2 n}, y_{2 n}, t\right), N\left(y_{2 n-1}, y_{2 n+1}, 2 t\right), N\left(y_{2 n-1}, y_{2 n}, t\right)\right\}\right)$

$\leq r^{\prime}\left(\max \left\{0, N\left(y_{2 n-1}, y_{2 n}, t\right), N\left(y_{2 n}, y_{2 n+1}, t\right), N\left(y_{2 n-1}, y_{2 n}, t\right)\right\}\right)$.

If $\min \left\{M\left(y_{2 n-1}, y_{2 n}, t\right), M\left(y_{2 n}, y_{2 n+1}, t\right)\right\}=M\left(y_{2 n}, y_{2 n+1}, t\right)$

and

$\max \left\{N\left(y_{2 n-1}, y_{2 n}, t\right), N\left(y_{2 n}, y_{2 n+1}, t\right)\right\}=N\left(y_{2 n}, y_{2 n+1}, t\right)$

then, a contradiction.

So,

$M\left(y_{2 n}, y_{2 n+1}, t\right) \geq r\left(M\left(y_{2 n-1}, y_{2 n}, t\right)\right)>M\left(y_{2 n-1}, y_{2 n}, t\right)$

and

$N\left(y_{2 n}, y_{2 n+1}, t\right) \leq r^{\prime}\left(N\left(y_{2 n-1}, y_{2 n}, t\right)\right)<N\left(y_{2 n-1}, y_{2 n}, t\right)$.

Similarly,

$M\left(y_{2 n+1}, y_{2 n+2}, t\right)>M\left(y_{2 n}, y_{2 n+1}, t\right)$

and

$N\left(y_{2 n+1}, y_{2 n+2}, t\right)<N\left(y_{2 n}, y_{2 n+1}, t\right)$.

In general,

$M\left(y_{n+1}, y_{n}, t\right) \geq r\left(M\left(y_{n}, y_{n-1}, t\right)\right)>M\left(y_{n}, y_{n-1}, t\right)$

and

$N\left(y_{n+1}, y_{n}, t\right) \leq r^{\prime}\left(N\left(y_{n}, y_{n-1}, t\right)\right)<N\left(y_{n}, y_{n-1}, t\right)$.

Thus $\left\{M\left(y_{n+1}, y_{n}, t\right)\right\}$ and $\left\{N\left(y_{n+1}, y_{n}, t\right)\right\}$ are the increasing and decreasing sequences of positive real numbers in $[0$, 1] and tends to a limit $l \leq 1$ and $e \geq 0$ respectively,

If $l<1$, then $\lim _{n \rightarrow \infty} M\left(y_{n+1}, y_{n}, t\right)=r(l)>l$

and

if $e>0$, then $\lim _{n \rightarrow \infty} N\left(y_{n+1}, y_{n}, t\right)=r^{\prime}(e)<e$,

which is a contradiction.

Therefore, $l=1$ and $e=0$.

Now for any positive integer $p$,

$M\left(y_{n}, y_{n+p}, t\right) \geq \min \left\{M\left(y_{n}, y_{n+1}, t / p\right), M\left(y_{n+1}, y_{n+2}, t / p\right), \ldots, M\left(y_{n+p-1}, y_{n+p}, t / p\right)\right\}$ 
and

$N\left(y_{n}, y_{n+p}, t\right) \leq \max \left\{N\left(y_{n}, y_{n+1}, t / p\right), N\left(y_{n+1}, y_{n+2}, t / p\right), \ldots, N\left(y_{n+p-1}, y_{n+p}, t / p\right)\right\}$.

Taking limit $n \rightarrow \infty$, we get

$\lim _{\mathrm{n} \rightarrow \infty} M\left(y_{n}, y_{n+p}, t\right) \geq \min \{1,1, \ldots, 1\}=1$

and

$\lim _{n \rightarrow \infty} N\left(y_{n}, y_{n+p}, t\right) \leq \max \{0,0, \ldots, 0\}=0$.

So, $\lim _{n \rightarrow \infty} M\left(y_{n}, y_{n+p}, t\right)=1$ and $\lim _{n \rightarrow \infty} N\left(y_{n,} y_{n+p}, t\right)=0$.

Thus $\left\{y_{n}\right\}$ is a Cauchy sequence in $X$. By the completeness of $X,\left\{y_{n}\right\}$ converges to $z \in X$. Hence

$P x_{2 n} \rightarrow z, S T x_{2 n+1} \rightarrow z, Q x_{2 n+1} \rightarrow z, A B x_{2 n+2} \rightarrow z$.

Firstly, suppose that $P$ is continuous. Since $(P, A B)$ is semi-compatible, we get

$P A B x_{2 n+2} \rightarrow P z$ and $P A B x_{2 n+2} \rightarrow A B z$.

Since the limit in intuitionistic fuzzy metric space is unique, we get

$P z=A B z$

We prove that $P z=z$. Put $x=z, y=x_{2 n+1}$ in (3.5) and let $P z \neq z$. then

$M\left(P z, Q x_{2 n+1}, t\right) \geq r\left(\min \left\{M\left(S T x_{2 n+1}, P z, t\right), M\left(A B z, Q x_{2 n+1}, 2 t\right), M\left(A B z, S T x_{2 n+1}, t\right)\right\}\right)$

and

$N\left(P z, Q x_{2 n+1}, t\right) \leq r^{\prime}\left(\max \left\{N\left(S T x_{2 n+1}, P z, t\right), N\left(A B z, Q x_{2 n+1}, 2 t\right), N\left(A B z, S T x_{2 n+1}, t\right)\right\}\right)$.

Letting $n \rightarrow \infty$ and using (3.6) and (3.8), we get

$M(P z, z, t) \geq r(\min \{M(z, P z, t), M(P z, z, 2 t), M(P z, z, t)\})$

$\geq r(M(P z, z, t))>M(P z, z, t)$

and

$N(P z, z, t) \leq r^{\prime}(\max \{N(z, P z, t), N(P z, z, 2 t), N(P z, z, t)\})$

$\leq r^{\prime}(N(P z, z, t))<N(P z, z, t)$

which is a contradiction and hence $z=P z=A B z$.

Put $x=B z$ and $y=x_{2 n+1}$ in (3.5) and as $B P=P B, A B=B A$ so we have

$M\left(P B z, Q x_{2 n+1}, t\right) \geq r\left(\min \left\{M\left(S T x_{2 n+1}, P B z, t\right), M\left(A B B z, Q x_{2 n+1}, 2 t\right), M\left(A B B z, S T x_{2 n+1}, t\right)\right\}\right)$

and

$N\left(P B z, Q x_{2 n+1}, t\right) \leq r^{\prime}\left(\max \left\{N\left(S T x_{2 n+1}, P B z, t\right), N\left(A B B z, Q x_{2 n+1}, 2 t\right), N\left(A B B z, S T x_{2 n+1}, t\right)\right\}\right)$.

Letting $n \rightarrow \infty$ and using (3.6), we get

$M(B z, z, t) \geq r(\min \{M(z, B z, t), M(B z, z, 2 t), M(B z, z, t)\})$

$\geq r(M(B z, z, t))>M(B z, z, t)$

and

$N(B z, z, t) \leq r^{\prime}(\max \{N(z, B z, t), N(B z, z, 2 t), N(B z, z, t)\})$

$\leq r^{\prime}(N(B z, z, t))<N(B z, z, t)$,

which is a contradiction and we get $B z=z$ and so $z=A B z=A z$.

Therefore $P z=A z=B z=z$.

Since $P(X) \subset S T(X)$ there exists $u \in X$ such that $z=P z=S T u$.

Put $x=x_{2 n}, y=u$ in equation (3.5), we get

$M\left(P x_{2 n}, Q u, t\right) \geq r\left(\min \left\{M\left(S T u, P x_{2 n}, t\right), M\left(A B x_{2 n}, Q u, 2 t\right), M\left(A B x_{2 n}, S T u, t\right)\right\}\right)$ 
American Research Journal of Mathematics, Volume 1, Issue 2, April 2015 ISSN 2378-704X

and

$N\left(P x_{2 n}, Q u, t\right) \leq r^{\prime}\left(\max \left\{N\left(S T u, P x_{2 n}, t\right), N\left(A B x_{2 n}, Q u, 2 t\right), N\left(A B x_{2 n}, S T u, t\right)\right\}\right)$.

Letting $n \rightarrow \infty$ and using (3.6), we get

$M(z, Q u, t) \geq r(\min \{M(z, z, t), M(z, Q u, 2 t), M(z, z, t)\})$

$\geq r(M(z, Q u, 2 t))>M(z, Q u, 2 t)$

and

$N(z, Q u t) \leq r^{\prime}(\max \{N(z, z, t), N(z, Q u, 2 t), N(z, z, t\}))$

$\leq r^{\prime}(N(z, Q u, 2 t))<N(z, Q u, 2 t)$,

which is a contradiction by Lemma 2.2 and we get, $Q u=z=S T u$.

Since $(Q, S T)$ is weak- compatible, we have $S T Q u=Q S T u$ i.e. $S T z=Q z$.

Put $x=x_{2 n}, y=z$ in (3.5), we get

$M\left(P x_{2 n}, Q z, t\right) \geq r\left(\min \left\{M\left(S T z, P x_{2 n}, t\right), M\left(A B x_{2 n}, Q z, 2 t\right), M\left(A B x_{2 n}, S T z, t\right)\right\}\right)$

and

$N\left(P x_{2 n}, Q z, t\right) \leq r^{\prime}\left(\max \left\{N\left(S T z, P x_{2 n}, t\right), N\left(A B x_{2 n}, Q z, 2 t\right), N\left(A B x_{2 n}, S T z, t\right)\right\}\right)$.

Letting $n \rightarrow \infty$ and using (3.6), we get

$M(z, Q z, t) \geq r(\min \{M(Q z, z, t), M(z, Q z, 2 t), M(z, Q z, t)\})$

$\geq r(M(z, Q z, t))>M(z, Q z, t)$

and

$N(z, Q z, t) \leq r^{\prime}(\max \{N(Q z, z, t), N(z, Q z, 2 t), N(z, Q z, t)\})$

$\leq r^{\prime}(N(z, Q z, t))<N(z, Q z, t)$,

which is a contradiction and we get $Q z=z$ and so $S T z=Q z=z$.

Put $x=x_{2 n}$ and $y=T z$ in (3.5), we get

$M\left(P x_{2 n}, Q T z, t\right) \geq r\left(\min \left\{M\left(S T T z, P x_{2 n}, t\right), M\left(A B x_{2 n}, Q T z, 2 t\right), M\left(A B x_{2 n}, S T T z, t\right)\right\}\right)$

and

$N\left(P x_{2 n}, Q T z, t\right) \leq r^{\prime}\left(\max \left\{N\left(S T T z, P x_{2 n}, t\right), N\left(A B x_{2 n}, Q T z, 2 t\right), N\left(A B x_{2 n}, S T T z, t\right)\right\}\right)$

As $Q T=T Q$ and $S T=T S$, we have $Q T z=T Q z=T z$ and $S T(T z)=T(S T z)=T z$.

Letting $n \rightarrow \infty$, we get

$M(z, T z, t) \geq r(\min \{M(T z, z, t), M(z, T z, 2 t), M(z, T z, t)\})$

$\geq r(M(z, T z, t))>M(z, T z, t)$

and

$N(z, T z, t) \leq r^{\prime}(\max \{N(T z, z, t), N(z, T z, 2 t), N(z, T z, t)\})$

$\leq r^{\prime}(N(z, T z, t))<N(z, T z, t)$

which is a contradiction and we get $T z=z$.

Now $S T z=T z=z$ implies $S z=z$.

By (3.10), we have $S z=T z=Q z=z$.

Combining (3.9) and (3.10), we get $A z=B z=P z=Q z=S z=T z=z$.

Hence, $z$ is a common fixed point of $A, B, P, Q, S$ and $T$.

Secondly, suppose that $A B$ is continuous.

Since $A B$ is continuous and $(P, A B)$ is semi-compatible, we get 
$A B P x_{2 n} \rightarrow A B z,(A B)^{2} x_{2 n} \rightarrow A B z, P A B x_{2 n} \rightarrow A B z$.

Thus $\lim _{n \rightarrow \infty} A B P x_{2 n}=\lim _{n \rightarrow \infty} P A B x_{2 n}=A B z$.

Put $x=A B x_{2 \mathrm{n}}, y=x_{2 n+1}$ in (3.5) and assuming $A B z \neq z$, we get

$M\left(P A B x_{2 n}, Q x_{2 n+1}, t\right) \geq r\left(\min \left\{M\left(S T x_{2 n+1}, P A B x_{2 n}, t\right), M\left((A B)^{2} x_{2 n}, Q x_{2 n+1}, 2 t\right)\right.\right.$,

$\left.\left.M\left((A B)^{2} x_{2 n}, S T x_{2 n+1}, t\right)\right\}\right)$

and

$N\left(P A B x_{2 n}, Q x_{2 n+1}, t\right) \leq r^{\prime}\left(\max \left\{N\left(S T x_{2 n+1}, P A B x_{2 n}, t\right), N\left((A B)^{2} x_{2 n}, Q x_{2 n+1}, 2 t\right)\right.\right.$,

$\left.\left.N\left((A B)^{2} x_{2 n}, S T x_{2 n+1}, t\right)\right\}\right)$.

Letting $n \rightarrow \infty$ and using (3.11), we get

$M(A B z, z, t) \geq r(\min \{M(z, A B z, t), M(A B z, z, 2 t), M(A B z, z, t)\})$

$\geq r(M(A B z, z, t))>M(A B z, z, t)$

and

$N(A B z, z, t) \leq r^{\prime}(\max \{N(z, A B z, t), N(A B z, z, 2 t), N(A B z, z, t)\})$

$\leq r^{\prime}(N(A B z, z, t))<N(A B z, z, t)$,

which is a contradiction and we get $\mathrm{ABz}=\mathrm{z}$.

Put $x=z, y=x_{2 n+1}$ in (3.5), we get

$M\left(P z, Q x_{2 n+1}, t\right) \geq r\left(\min \left\{M\left(S T x_{2 n+1}, P z, t\right), M\left(A B z, Q x_{2 n+1}, 2 t\right), M\left(A B z, S T x_{2 n+1}, t\right)\right\}\right)$

and

$N\left(P z, Q x_{2 n+1}, t\right) \leq r^{\prime}\left(\max \left\{N\left(S T x_{2 n+1}, P z, t\right), N\left(A B z, Q x_{2 n+1}, 2 t\right), N\left(A B z, S T x_{2 n+1}, t\right)\right\}\right)$.

Letting $n \rightarrow \infty$ and using (3.6), we get

$M(P z, z, t) \geq r(\min \{M(z, P z, t), M(z, z, 2 t), M(z, z, t)\})$

$\geq r(M(z, P z, t))>M(P z, z, t)$

and

$N(P z, z, t) \leq r^{\prime}(\max \{N(z, P z, t), N(z, z, 2 t), N(z, z, t)\})$

$\leq r^{\prime}(N(z, P z, t))<N(P z, z, t)$,

which gives $P z=z$. Hence, $P z=z=A B z$.

Thus $A B z=z$ gives $A z=z$ and so $A z=B z=P z=z$.

That is, $A z=B z=P z=Q z=S z=T z=z$.

Hence $z$ is a common fixed point of $A, B, P, Q, S$ and $T$ in this case also.

Uniqueness: Let $z_{1}$ be another common fixed point of $A, B, P, Q, S$ and $T$.

Then $A z_{1}=B z_{1}=P z_{1}=Q z_{1}=S z_{1}=T z_{1}=z_{1}$, assuming $z \neq z_{1}$. Put $x=z, y=z_{1}$ in (3.5), we get

$M\left(P z, Q z_{1}, t\right) \geq r\left(\min \left\{M\left(S T z_{1}, P z, t\right), M\left(A B z, Q z_{1}, 2 t\right), M\left(A B z, S T z_{1}, t\right)\right\}\right)$

$M\left(z, z_{1}, t\right) \geq r\left(\min \left\{M\left(z_{1}, z, t\right), M\left(z, z_{1}, 2 t\right), M\left(z, z_{1}, t\right)\right\}\right)$

$\geq r\left(M\left(z, z_{1}, t\right)\right)>M\left(z, z_{1}, t\right)$

and

$N\left(P z, Q z_{1}, t\right) \leq r^{\prime}\left(\max \left\{N\left(S T z_{1}, P z, t\right), N\left(A B z, Q z_{1}, 2 t\right), N\left(A B z, S T z_{1}, t\right)\right\}\right)$

$N\left(z, z_{1}, t\right) \leq r^{\prime}\left(\max \left\{N\left(z_{1}, z, t\right), N\left(z, z_{1}, 2 t\right), N\left(z, z_{1}, t\right)\right\}\right)$

$\leq r^{\prime}\left(N\left(z, z_{1}, t\right)\right)<N\left(z, z_{1}, t\right)$

which is a contradiction. Hence $z=z_{1}$ and so $z$ is the unique common fixed point of $A, B, P, Q, S$ and $T$. 
Corollary 3.1: Let $A, S, P$ and $Q$ be self-mappings on a complete intuitionistic fuzzy metric space $\left(X, M, N,{ }^{*}, \diamond\right)$ satisfying:

$P(X) \subset S(X), Q(X) \subset A(X)$,

either $P$ or $A$ is continuous,

$(P, A)$ is semi-compatible and $(Q, S)$ is weak-compatible,

for all $x, y \in X$ and $t>0$

$M(P x, Q y, t) \geq \min \{M(S y, P y, t), M(A x, Q y, 2 t), M(A x, S y, t)\}$

and

$N(P x, Q y, t) \leq \max \{N(S y, P y, t), N(A x, Q y, 2 t), N(A x, S y, t)\}$

Then $A, S, P$ and $Q$ have a unique common fixed point in $X$.

Corollary 3.2: Let $A, B, S, T, P$ and $Q$ be self-mappings on a complete intuitionistic fuzzy metric space $(X, M, N$, $*, \diamond)$ satisfying

$P(X) \subset S T(X), Q(X) \subset A B(X)$,

$A B=B A, S T=T S, P B=B P$ and $Q T=T Q$,

either $P$ or $A B$ is continuous,

$(P, A B)$ is semi-compatible and $(Q, S T)$ is weak-compatible,

for all $x, y \in X$ and $t>0$

$M(P x, Q y, t) \geq \min \{M(S T y, P y, t), M(A B x, Q y, 2 t), M(A B x, S T y, t)\}$

and

$N(P x, Q y, t) \leq \max \{N(S T y, P y, t), N(A B x, Q y, 2 t), N(A B x, S T y, t)\}$.

Then $A, B, S, T, P$ and $Q$ have a unique common fixed point in $X$.

\section{CONCLUSION}

The present paper extended and generalized various known fixed point theorems in the literature in the setting of fuzzy and intuitionistic fuzzy metric spaces.

\section{REFERENCES}

[1] L. A. Zadeh, Fuzzy sets, Inform. and Control, 89(1965), 338-358.

[2] J. Kramosil and J. Michalek, Fuzzy metric and statistical metric spaces, Kybernetica 11(1975), 326-334.

[3] A.George and P. Veeramani, On some results in fuzzy metric spaces, Fuzzy Sets and Systems, 64 (1994), 395-399.

[4] J. H. Park, Intuitionistic fuzzy metric spaces, Chaos Solitions \& Fractals, 22 (2004), 1039-1046.

[5] C. Alaca, D. Turkoglu and C. Yildiz, Fixed points in intuitionistic fuzzy metric spaces, Choas Solitons \& Fractals, 29(5)(2006), 1073-1078.

[6] D. Turkoglu, C. Alaca, Y. J. Cho., and C. Yildiz, Common fixed points theorems in intuitionistic fuzzy metrics paces, J. Appl. Math. \& Computing, 22 (2006), 411-424.

[7] G. Jungck, Commuting mappings and fixed points, Amer. Math. Monthly, 83 (1976), 261-263.

[8] B. Singh and M. S. Chauhan, Common fixed points of compatible maps in fuzzy metric spaces, Fuzzy Sets and Systems, 115(2000), 471-475.

[9] B. Schweizer and A. Sklar, Statistical metric spaces, Pacific J. Math., 10 (1960), 314-334.

[10] B. Singh and S. Jain, Semi-compatibility, compatibility and fixed point theorems in fuzzy metric space, Journal of Chungecheong Math. Soc., 18(1)(2005), 1-22.

[11] J. S. Park and S. Y. Kim, Common fixed point and example in intuitionistic fuzzy metric space, J. K. I. I. S., 18(4)(2008), $524-529$. 